\title{
Construction of Confidence Interval of the Parameter in Von Mises Distribution using Bootstrap Methods
}

\author{
Nor Hafizah Moslim ${ }^{1,2}$, Yong Zulina Zubairi ${ }^{3,{ }^{*}}$, Abdul Ghapor Hussin ${ }^{4}$, Siti Fatimah Hassan ${ }^{3}$ and \\ Nurkhairany Amyra Mokhtar ${ }^{4}$ \\ ${ }^{1}$ Institute of Advanced Studies, University of Malaya, 50603 Kuala Lumpur, \\ ${ }^{2}$ Centre for Mathematical Sciences, Universiti Malaysia Pahang, 26300 Gambang, Kuantan, Pahang, \\ ${ }^{3}$ Center of Foundation Studies in Sciences, University of Malaya, 50603 Kuala Lumpur, \\ ${ }^{4}$ Faculty of Defence Sciences \& Technology, National Defence University of Malaysia,
} Kem Sg Besi, 57000 Kuala Lumpur

\begin{abstract}
Bootstrap method is a computer-based technique for making certain kind of statistical inferences which can simplify the often intricate calculations of traditional statistical theory. Recently, bootstrapping has been widely used for the parameter estimation of linear data. In this paper, we consider bootstrapping methods in the construction of the confidence interval of concentration parameter, $\kappa$ for the von Mises distribution. The performances of confidence interval based on percentile bootstrap, bootstrap- $t$ and calibration bootstrap are evaluated via simulation study. The numerical results found that confidence interval based on the calibration bootstrap is good in terms of coverage probability. Meanwhile, confidence interval based on the bootstrap- $t$ method has a shorter expected length. The confidence intervals were illustrated using daily wind direction data recorded at maximum wind speed for four stations in Malaysia. From point estimates of the concentration parameter and the respective confidence interval, we note that the method works well for a wide range of $\kappa$ values. The implication of the study is that confidence interval of the concentration parameter can be obtained using bootstrap as it provides good estimates.
\end{abstract}

Keywords: bootstrap- $t$; calibration bootstrap; concentration parameter; percentile bootstrap; von Mises distribution

\section{INTRODUCTION}

Bootstrap method is a computer-based technique for making certain kind of statistical inferences which can simplify the often intricate calculations of traditional statistical theory (Efron \& Tibshirani, 1993). It substitutes considerable amount of computation in place of the theoretical analysis. This method was introduced as a nonparametric device for estimating standard errors and biases and has been in use since 1980s. DiCiccio \& Efron (1996) described the types of bootstrap used for confidence interval. Efron and Tibshirani (1986) explained the bootstrap estimate of standard error, measures of statistical accuracy and bootstrap confidence intervals. Other disciplines use the bootstrap methods in analysing their data and these include in biology (Fung, 1996; Caccone et al., 1996), physics (Zamolodchikov, 1996), economics (Zaher \& Featherstone, 2010), geography (Yan et al., 2015), medicine (Dwivedi et al., 2017) and hospitality and tourism (Azdel et al., 2015).

Statistical data can be classified according to their distributional topologies. A linear data set can be represented on a straight line and for circular data, they can be represented by the circumference of a circle. For circular 
data, they are commonly measured in the range of $\left[0^{\circ}, 360^{\circ}\right)$ degrees or $[0,2 \pi)$ radian. It is worthwhile to note that statistical theories for straight line and circle are very different from one to another because the circle is a closed curve. Circular or directional data can be found in the area of meteorology, medicine, geology, image analysis and astronomy (Mardia, 1972; Mardia \& Jupp, 2000).

Von Mises distribution is said to be the most useful distribution in describing circular random variable. The density function is given as;

$$
f(\theta ; \mu, \kappa)=\frac{1}{2 \pi I_{0}(\kappa)} \exp (\kappa \cos (\theta-\mu))
$$

where $0 \leq \mu<2 \pi$ and $\kappa>0$ are the parameters. $I_{0}(\kappa)$ is the modified Bessel function of order zero and can be defined as;

$$
I_{0}(\kappa)=\frac{1}{2 \pi} \int_{0}^{2 \pi} \exp (\kappa \cos (\theta-\mu)) d \theta
$$

This is a continuous probability distribution and as $\kappa$ approaches $\mathrm{o}$, the distribution converges to the uniform distribution. Meanwhile, as $\kappa$ increase, the distribution converges to the point distribution concentrated in the direction $\mu$. Thus, it will approach the normal distribution with the mean $\mu_{0}$ and variance $\frac{1}{\kappa}$ (Fisher, 1993; Mardia \& Jupp, 2000). Since then, von Mises distribution can also be called as Circular Normal Distribution as it has the similarities with the normal distribution on the real line (Fisher, 1993).

In data analysis, confidence interval is often used as they combine both point estimate and hypothesis testing into a single inferential statement. In other words, confidence interval gives an estimated range of values which is likely to include an unknown population parameter with a specified probability within that interval. A number of studies were done to approximate confidence interval for the concentration parameter of von Mises distribution including those using bootstrap (Stephens, 1969; Khanabsakdi, 1995; Hassan et al., 2014).

\section{METHODS}

One early method of obtaining confidence interval for concentration parameter is using the percentile bootstrap method (Fisher, 1993). This approach is further improved using bootstrap-t (Hassan et al., 2014). However, the bootstrap- $t$ method only limits to the second-order accuracy and the algorithm can be numerically unstable (DiCiccio \& Efron, 1996). Thus, to ensure good coverage accuracy and overall expected length, we propose a calibration bootstrap method which improves to the third-order accuracy. In this study, confidence interval for the concentration parameter based on calibration bootstrap will be proposed. The confidence interval based on the percentile bootstrap and bootstrap- $t$ will be considered as well. The performances of the confidence intervals which are coverage probability and expected length will be evaluated via simulation study (Letson \& McCullough, 1998).

\section{A. Confidence Interval based on Percentile Bootstrap}

Among all the bootstrap methods, percentile bootstrap is often and widely used in confidence interval. For circular statistics, explanations and guidance in constructing the confidence interval for the concentration parameter based on percentile bootstrap can be found in Fisher (1993). Following are the steps in performing the percentile bootstrap method for the simulation purpose:

\section{Step a: Resampling}

Simulate $n$ values of $\theta_{i}$ from the $\operatorname{VM}(\hat{\mu}, \hat{\kappa})$ where

$$
0 \leq \theta_{i}<2 \pi \text { and } i=1,2, \ldots, n \text {. }
$$

\section{Step b: Bootstrap parameter estimate}

Estimate the bootstrap parameter for the bootstrap samples from step a and label it as $\hat{\kappa}_{1}$.

\section{Step c: Repetition}

Repeat step a and $\mathbf{b}$ to obtain $B$ bootstrap parameter estimates, $\hat{\kappa}_{1}, \hat{\kappa}_{2}, \ldots, \hat{\kappa}_{B}$.

\section{Step d: Confidence Interval}

(i) Arrange the bootstrap parameter estimates, $\hat{\kappa}_{1}, \hat{\kappa}_{2}, \ldots, \hat{\kappa}_{B}$ in increasing order:

$\hat{\kappa}_{(1)} \leq \hat{\kappa}_{(2)} \leq \ldots \leq \hat{\kappa}_{(B)}$ 
(ii) The $100(1-\alpha) \% \mathrm{CI}$ for $\kappa$ is given as:

$$
\begin{aligned}
& \left(\hat{\kappa}_{(l+1)}, \hat{\kappa}_{(m)}\right) \text { where } \\
& l=\left\lfloor\frac{1}{2} B \alpha+\frac{1}{2}\right\rfloor \text { and } m=B-1
\end{aligned}
$$

\section{B. Confidence Interval based on Bootstrap-t}

Bootstrap- $t$ was proved to be the best method for confidence interval for the concentration parameter in terms of coverage probability (Hassan et al., 2014). By having the smaller coverage error, it could outperform the percentile bootstrap and bias corrected and accelerated bootstrap (BCA) method (Hall, 1986). The following steps are carried out for the simulation purpose:

\section{Step a: Resampling}

Simulate $n$ values of $\theta_{i}$ from the $\operatorname{VM}(\hat{\mu}, \hat{\kappa})$ where $0 \leq \theta_{i}<2 \pi$ and $i=1,2, \ldots, n$.

\section{Step b: Bootstrap parameter estimate}

(i) Estimate the bootstrap parameter for the bootstrap samples from step a and label it as $\hat{\kappa}_{1}$.

(ii) Calculate the standard error (SE) for the estimated bootstrap parameter and label it as $\hat{S}_{1}$ where $\quad \operatorname{var}\left(\hat{\kappa}_{1}\right)=\frac{1}{n\left(1-\frac{\bar{R}_{1}}{\hat{\kappa}_{1}}-\bar{R}_{1}^{2}\right)}$, $\bar{R}_{1}=\sqrt{\bar{C}_{1}^{2}+\bar{S}_{1}^{2}}, \quad \bar{C}_{1}=\frac{1}{n} \sum_{i=1}^{n} \cos \theta_{i} \quad$ and $\bar{S}_{1}=\frac{1}{n} \sum_{i=1}^{n} \sin \theta_{i}$

(iii) Calculate the $t$-value given by

$$
t_{1}=\frac{\hat{\kappa}_{1}-\hat{\kappa}}{\hat{S}_{1}}
$$

where $\hat{S}_{1}$ is the estimated standard error of $\hat{\kappa}_{1}$ based on the data in step a.

\section{Step c: Repetition}

Repeat step a and $\mathbf{b}$ to obtain $B$ bootstrap $t$-values, $t_{1}, t_{2}, \ldots, t_{B}$ of the concentration parameter.

\section{Step d: Confidence Interval}

(i) Arrange the $t$-values, $t_{1}, t_{2}, \ldots, t_{B}$ in increasing order: $t_{(1)} \leq t_{(2)} \leq \ldots \leq t_{(B)}$

(ii) The $100(1-\alpha) \% \mathrm{CI}$ for $\kappa$ is given as:

$$
\left(\hat{\kappa}-t_{(1-\alpha)} S, \hat{\kappa}-t_{(\alpha)} S\right)
$$

where $t_{(1-\alpha)}$ is $1-\alpha$ percentile of $t_{b}$ values, $t_{(\alpha)}$ is $\alpha$ percentile of $t_{b}$ values and $S$ is the estimated standard error for $\hat{\kappa}$.

\section{Confidence Interval based on Calibration Bootstrap}

Calibration is a bootstrap resampling technique that performs a second bootstrap loop. Although it is computationally intensive, this can be easily overcomed with the advancement of technology and supercomputing facilities (Lv et al., 2017). DiCiccio \& Efron (1996) explained that this method was much more stable and lead to the accurate intervals.

Let $p(\hat{\lambda})=\operatorname{Prob}\left\{\theta \leq \hat{\theta}_{\hat{\lambda}}\right\}=\alpha$. Once the value of $\hat{\lambda}$ is obtained and if the procedure is calibrated correctly, the value of $\lambda=\alpha$ is achieved.

Let $\hat{p}(\lambda)=\operatorname{Prob}_{*}\left\{\hat{\theta} \leq \hat{\theta}_{\lambda}^{*}\right\}$ be the bootstrap estimate of $p(\lambda)$ where “*” refers to the bootstrap sampling and $\hat{\theta}$ is fixed. Generate a number of bootstrap samples then compute $\hat{\theta}_{\lambda}^{*}$ for each one and record the proportion of times that $\hat{\theta} \leq \hat{\theta}_{\lambda}^{*}$. By using the same bootstrap samples, the process is repeated for a range of $\lambda$ values that includes the nominal value $\alpha$. The value of $\lambda$ that satisfy $\hat{p}(\lambda)=\alpha$ is denoted by $\hat{\lambda}_{\alpha}$.

The following steps describe the calibration bootstrap method:

1. Generate $n$ values of $\theta_{i}$ from the $\operatorname{VM}(\hat{\mu}, \hat{\kappa})$ where $0 \leq \theta_{i}<2 \pi$ and $i=1,2, \ldots, n$. 
2. Estimate the bootstrap parameter for the bootstrap samples from step (1) and label it as $\hat{\kappa}_{1}$.

3. Repeat step (1) and (2) to obtain $B$ bootstrap parameter estimates; $\hat{\kappa}_{b}$ where $b=1,2, \ldots, B$.

4. For each bootstrap samples, compute a $\lambda$ level confidence point $\hat{\theta}_{\lambda}^{*}(b)$ for a range of $\lambda$ values.

5. Get the value of $\hat{p}(\lambda)=\#\left\{\hat{\theta} \leq \hat{\theta}_{\lambda}^{*}(b)\right\} / B$ for each $\lambda$.

6. Find the value of $\lambda$ that satisfy $p(\lambda)=\alpha$.

\section{Simulation Study}

Simulation study were conducted for three different sample sizes, $n=30,50$ and 100 with six values of concentration parameter, $\kappa=1,2,3,5,10$ and 15 , respectively. Without loss of generality, the mean direction value, $\mu$ will be assumed as 0 . The significance level for the percentile bootstrap and bootstrap- $t$ is set at $\alpha=0.05$ meanwhile for the calibration bootstrap method is $\alpha=0.04$. This has been evaluated previously to be the suitable value of $\alpha$ to get the probability of 0.95. The number of bootstrap replications, $B$ for each simulation is set at 100 (Efron \& Tibshirani, 1993). Let $s$ be the number of simulation studies and it was repeated for 360 times. Two indicators to determine the best method in constructing intervals were calculated as follows:

(a) coverage probability $=\mathrm{m} / \mathrm{s}$ where $\mathrm{m}$ is the number of true value that fall within the confidence interval.

(b) expected length = upper limit - lower limit.

Coverage probability is the proportion number that the confidence interval contains the true value of concentration parameter for each method. The confidence level considered in this study is $95 \%$. Thus, the best result is measured through the coverage probability value that is close to 0.95 . Expected length is the class size of a confidence interval. It is another indicator to determine the best method of constructing the confidence interval. The best and efficient method will give the shortest expected length.

\section{RESULTS AND DISCUSSIONS}

Table 1 and Table 2 display the results of coverage probability and expected length for all values of sample size, $n$ and concentration parameter, $\kappa$ for each method, respectively.

Each method is labelled as follows:

\section{(A) B1 - PERCENTILE BOOTSTRAP (B) B2 - BOOTSTRAP-T \\ (C) B3 - CALIBRATION BOOTSTRAP}

Table 1. Coverage probability for sample size, $n=30,50$ and 100 and concentration parameter, $\kappa=1,2,3,5,10$ and 15

\begin{tabular}{|c|c|c|c|c|}
\hline \multirow{2}{*}{$n$} & \multirow{2}{*}{$\kappa$} & \multicolumn{3}{|c|}{ Method } \\
\hline & & B1 & B2 & B3 \\
\hline \multirow{6}{*}{30} & 1 & 0.889 & 0.939 & 0.938 \\
\hline & 2 & 0.889 & 0.942 & 0.926 \\
\hline & 3 & 0.894 & 0.911 & 0.921 \\
\hline & 5 & 0.886 & 0.925 & 0.917 \\
\hline & 10 & 0.853 & 0.917 & 0.916 \\
\hline & 15 & 0.869 & 0.922 & 0.916 \\
\hline \multirow{6}{*}{50} & 1 & 0.914 & 0.931 & 0.946 \\
\hline & 2 & 0.919 & 0.906 & 0.940 \\
\hline & 3 & 0.897 & 0.939 & 0.937 \\
\hline & 5 & 0.881 & 0.931 & 0.934 \\
\hline & 10 & 0.919 & 0.917 & 0.930 \\
\hline & 15 & 0.908 & 0.933 & 0.930 \\
\hline \multirow{6}{*}{100} & 1 & 0.914 & 0.956 & 0.952 \\
\hline & 2 & 0.939 & 0.931 & 0.946 \\
\hline & 3 & 0.922 & 0.961 & 0.948 \\
\hline & 5 & 0.931 & 0.936 & 0.944 \\
\hline & 10 & 0.878 & 0.944 & 0.944 \\
\hline & 15 & 0.900 & 0.944 & 0.943 \\
\hline
\end{tabular}

As sample size increases in Table 1, the coverage probability approaches the target value (0.95) for each concentration parameter, $\kappa$. B1 gives consistently lower coverage probability than the target value which leads to the poorest performance method. As sample size, $n$ and concentration parameter, $\kappa$ increases, the coverage probability using the B3 method is very close to the target value. Thus, B3 is the 
best method based on the performance of coverage probability.

From the results obtained and displayed in Table 2, it can be seen that the expected length value increases as the concentration parameter, $\kappa$ increases for each sample size, $n$. For each concentration parameter, $\kappa$ increase in the sample size, $n$ results in a decrease of the expected length for all methods. The expected length value of B1 method consistently gives larger value. Meanwhile, the expected length value of $\mathrm{B}_{2}$ and $\mathrm{B}_{3}$ methods are very close to each other with B2 consistently smaller. Thus, we can infer that B2 is the superior method based on this performance.

Table 2. Expected length for sample size,

I. $n=30,50$ and 100 and concentration parameter,

$$
\text { II. } \kappa=1,2,3,5,10 \text { and } 15
$$

\begin{tabular}{|c|c|c|c|c|}
\hline \multirow{2}{*}{$n$} & \multirow{2}{*}{$\kappa$} & \multicolumn{3}{|c|}{ Method } \\
\hline & & B1 & B2 & B3 \\
\hline \multirow{6}{*}{30} & 1 & 1.4258 & 1.1320 & 1.1648 \\
\hline & 2 & 2.4429 & 1.7270 & 1.7637 \\
\hline & 3 & 3.8603 & 2.6446 & 2.7262 \\
\hline & 5 & 6.2738 & 4.4233 & 4.5853 \\
\hline & 10 & 14.0267 & 10.0058 & 10.2404 \\
\hline & 15 & 19.8517 & 14.3233 & 14.6374 \\
\hline \multirow{6}{*}{50} & 1 & 1.0151 & 0.9053 & 0.9333 \\
\hline & 2 & 1.5808 & 1.3093 & 1.3466 \\
\hline & 3 & 2.5966 & 2.0558 & 2.1199 \\
\hline & 5 & 4.6363 & 3.7672 & 3.8667 \\
\hline & 10 & 9.6311 & 7.7536 & 7.9798 \\
\hline & 15 & 14.0911 & 11.8226 & 11.4588 \\
\hline \multirow{6}{*}{100} & 1 & 0.6901 & 0.6521 & 0.6725 \\
\hline & 2 & 1.0538 & 0.9698 & 1.0022 \\
\hline & 3 & 1.6311 & 1.4605 & 1.4985 \\
\hline & 5 & 2.9260 & 2.6487 & 2.7431 \\
\hline & 10 & 5.4827 & 4.8788 & 5.0030 \\
\hline & 15 & 9.2079 & 8.2549 & 8.5628 \\
\hline
\end{tabular}
probability in Table 1, B3 is the superior method. Meanwhile, based on the expected length, method B2 performs better than method B1 and B3.

\section{ILLUSTRATIVE EXAMPLE}

As an illustration of the proposed method, daily wind direction data (in radian) recorded at maximum wind speed (in $\mathrm{m} / \mathrm{s}$ ) for four stations in Malaysia were considered. These data were collected from the year of 2013 to 2017 at an altitude of $2.1 \mathrm{~m}$ to $16.1 \mathrm{~m}$. A total of 80 data points was obtained from each stations which are located at peninsular and east Malaysia. For the peninsular Malaysia, the stations are located at west coast and east coast regions. For the west coast region, the stations are located at Alor Setar and Kuala Lumpur International Airport (KLIA) meanwhile for the east coast region, the station is located at Kuala Terengganu. For east Malaysia, a station located at Kota Kinabalu was considered. Figure 1 shows the locations of all stations. All data were obtained from Malaysian Meteorological Department.

Table 3 shows the confidence intervals and expected lengths for concentration parameter of wind direction based on the three methods for four stations. 


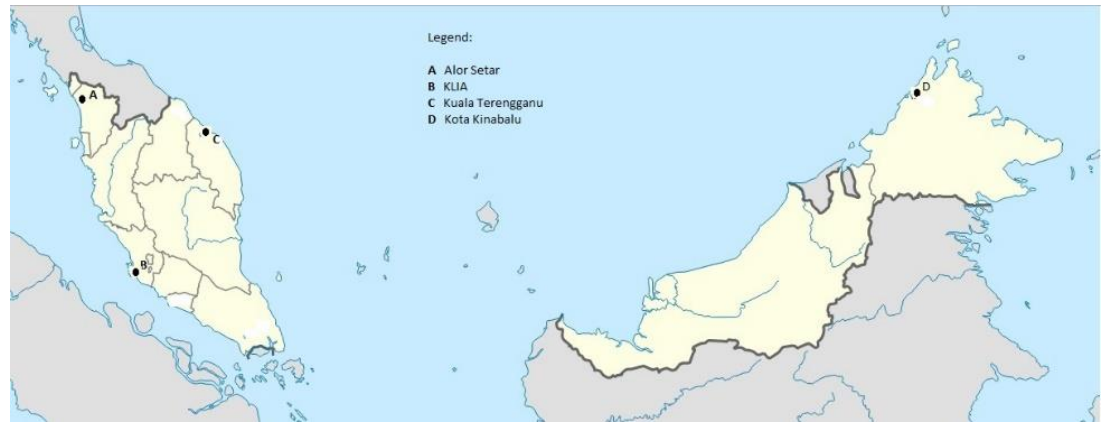

Figure 1. Malaysia Map (ref: ms.wikipedia.org/wiki/Fail:Malaysia_location_map.svg)

For each station, the estimation concentration parameter, $\hat{\kappa}$ is calculated. All estimation values are located in the range of confidence interval. From the estimation values of all stations, Kuala Terengganu recorded the highest estimation value which means that the wind direction at east coast region is less scattered and more concentrated. The wind direction at KLIA station which located at west coast region is more scattered and more dispersed since it recorded lowest value of estimation concentration parameter.
By comparing on the expected length, all results are similar with the findings from the simulation studies. For all stations, B1 method (percentile bootstrap) consistently give largest expected length value. The expected length values of B2 (bootstrap- $t$ ) and B3 (calibration bootstrap) methods are close to each other where $\mathrm{B}_{3}$ record slightly larger value than B2 methods.

Table 3. Confidence Interval (CI) for wind direction data recorded at maximum wind speed

\begin{tabular}{|c|c|c|c|c|c|}
\hline Region & Station & $\hat{\kappa}$ & Method & Confidence Interval & Expected Length \\
\hline \multirow{6}{*}{$\begin{array}{c}\text { West Coast, } \\
\text { Peninsular } \\
\text { Malaysia }\end{array}$} & \multirow{3}{*}{ Alor Setar } & \multirow{3}{*}{2.6272} & $\mathrm{~B} 1$ & $(2.1107,3.7782)$ & 1.6675 \\
\hline & & & B2 & $(2.0122,3.2587)$ & 1.2465 \\
\hline & & & $\mathrm{B} 3$ & $(1.9616,3.2587)$ & 1.2971 \\
\hline & \multirow{3}{*}{ KLIA } & \multirow{3}{*}{1.2270} & B1 & $(0.8810,1.9377)$ & 1.0567 \\
\hline & & & B2 & $(0.9554,1.7279)$ & 0.7725 \\
\hline & & & $\mathrm{B}_{3}$ & $(0.9174,1.7279)$ & 0.8105 \\
\hline \multirow{3}{*}{$\begin{array}{c}\text { East Coast, } \\
\text { Peninsular } \\
\text { Malaysia }\end{array}$} & \multirow{3}{*}{ Kuala Terengganu } & \multirow{3}{*}{$7 \cdot 3049$} & B1 & $(5.8782,11.2306)$ & $5 \cdot 3524$ \\
\hline & & & B2 & $(5.5606,9.4539)$ & 3.8933 \\
\hline & & & B3 & $(5.3673,9.4539)$ & 4.0866 \\
\hline \multirow{3}{*}{ East Malaysia } & \multirow{3}{*}{ Kota Kinabalu } & \multirow{3}{*}{2.3332} & B1 & $(1.8660,3.2501)$ & 1.3842 \\
\hline & & & $\mathrm{B} 2$ & $(1.8218,3.0174)$ & 1.1956 \\
\hline & & & B3 & $(1.7415,3.0174)$ & 1.2759 \\
\hline
\end{tabular}




\section{SUMMARY}

This article considers calibration bootstrap method in constructing the confidence interval of the concentration parameter, $\kappa$ for the von Mises distribution. The method is derived and compared with percentile bootstrap and bootstrap- $t$ methods. Two indicators; coverage probability and expected length were calculated in determining the best method. The coverage probability value is more influenced and often used in measuring the performance of confidence interval. Based on the results, calibration bootstrap method performs better compared to the other bootstrap methods. The methods were tested using real data set and the results aligned with the simulation results.

\section{ACKNOWLEDGMENT}

The authors wish to thank University of Malaya (BKo45-2016 and GBFoo6-2018) for the research grant in undertaking this research.

\section{REFERENCES}

Azdel, A.A., Bakhtiar, M.F.S., Kamaruddin, M.S.Y., Ahmad, N.A. \& Ahmat, N.C. 2014, An Investigation of Customers' Satisfaction as a Mediating Effect Between Hotel Customer Loyalty Antecedents and Behavioral Loyalty. Theory and Practice In Hospitality and Tourism Research: Proceedings of the 2nd International Hospitality \& Tourism Conference, 2nd-4th Sept2014, Penang, Malaysia.

Caccone, A., Moriyama, E.N., Gleason, J.M., Nigro, L. \& Powell, J.R. 1996, A Molecular Phylogeny for the Drosophila Melanogaster Subgroup and the Problem of Polymorphism Data. Molecular Biology and Evolution, 13(9), 1224-1232.

DiCiccio, T.J. \& Efron, B. 1996, Bootstrap Confidence Interval. Statistical Science, 11(3),189-228.

Dwivedi, A.K., Mallawaarachchi, I. \& Alvarado, L.A. 2017, Analysis of Small Sample Size Studies using Nonparametric Bootstrap Test with Pooled Resampling Method. Statistics in Medicine, 36(14), 2187-2205.

Efron, B. \& Tibshirani, R.J. 1986, Bootstrap Methods for Standard Errors, Confidence Intervals, and Other Measures of Statistical Accuracy. Statistical Science, 1(1), 54-77.

Efron, B. \& Tibshirani, R.J. 1993, An Introduction to the Bootstrap. London: Chapman \&Hall.

Fisher, N.I. 1993. Statistical Analysis of Circular Data. Cambridge University Press, Cambridge.

Fung, W.K. 1996, Are Convenience DNA Samples Significantly Different? Forensic Science International, 82(3)3, 233-241.

Hall, P. 1986, On the Bootstrap and Confidence Interval. The Annals of Statistics, 14(4),1431-1452.
Hassan, S.F., Zubairi, Y.Z., Hussin, A.G. \& Satari, S.Z. 2014, Some Confidence Intervals for Large Concentration Parameter in Von Mises Distribution. Pakistan Journal of Statistics, 30(2), 273-284.

Khanabsakdi, S. 1995-1996, Inferential Statistics for Concentration of Directional Data using the Chi Square Distribution. The Philippine Statistician, 44-45(1-8), 61-67.

Letson, D. \& McCullough, B.D. 1998, Better Confidence Intervals: The Double Bootstrap with No Pivot. American Journal of Agricultural Economics, 8o(3), 552-559.

Lv, X., Zhang, G. \& Xu, X. 2017. Bootstrap Calibrated Empirical Likelihood Confidence Intervals for the Difference between Two Gini Indexes. Journal of Economic Inequality15(2):195-216.

Mardia, K.V. \& Jupp, P.E. 200o, Directional Statistics. England: John Wiley \& Sons Ltd.

Mardia, K.V. 1972, Statistics of Directional Data. London: Academic Press.

Stephens, M.A. 1969. A Goodness-of-Fit Statistic for the Circle with some Comparisons. Biometrika, 56(1), 161-168.

Yan, N., Mei, C.L. \& Wang, N. 2015. A Unified Bootstrap Test for Local Patterns of Spatiotemporal Association. Environment and Planning A, 47(1), 227-242.

Zaher, M. \& Featherstone, A.M. 2010. Productive Efficiency in the Middle East and North Africa. Applied Economics, 42(7), 899-915.

Zamolodchikov, A. \& Zamolodchikov, A.L 1996, Conformal Bootstrap in Liouville Field Theory. Nuclear Physics B, 477(2), 577-605. 